Article

\title{
Fetal Megacystis: A New Morphologic, Immunohistological and Embriogenetic Approach
}

\author{
Lidia Puzzo ${ }^{1, *}$, Giuliana Giunta ${ }^{2}$, Rosario Caltabiano ${ }^{1}$, Antonio Cianci $^{2}$ and \\ Lucia Salvatorelli ${ }^{1}$
}

1 Department of Medical and Surgical Sciences and Advanced Technologies, G.F. Ingrassia, Azienda Ospedaliero-Universitaria "Policlinico-Vittorio Emanuele", Anatomic Pathology Section, School of Medicine, University of Catania, 95123 Catania, Italy; rosario.caltabiano@unict.it (R.C.); lucia.salvatorelli@unict.it (L.S.) Department of General Surgery and Medical Surgical Specialties, Department of Obstetrics and Gynecology-Policlinico Universitario G. Rodolico, University of Catania, 95123 Catania, Italy; giuntagiuliana.ct@gmail.com (G.G.); acianci@unict.it (A.C.)

* Correspondence: lipuzzo@unict.it; Tel.: +39-095-3782026; Fax: +39-095-3782023

Received: 23 September 2019; Accepted: 26 October 2019; Published: 28 November 2019

check for updates

\begin{abstract}
Congenital anomalies of the kidney and urinary tract (CAKUT) include isolated kidney malformations and urinary tract malformations. They have also been reported in Prune-Belly syndrome (PBS) and associated genetic syndromes, mainly 13, 18 and 21 trisomy. The AA focuses on bladder and urethral malformations, evaluating the structural and histological differences between two different cases of megacystis. Both bladders were examined by routine prenatal ultrasound screening and immunohistochemistry, comparing the different expression of smooth muscular actin (SMA), S100 protein and WT1c in megacystis and bladders of normal control from fetuses of XXI gestational age. Considering the relationship between the enteric nervous system and urinary tract development, the AA evaluated S100 and WT1c expression both in bladder and bowel muscular layers. Both markers were not expressed in the bladder and bowel of PBS associated with anencephaly. In conclusion, megacystis could be considered only a macroscopic definition, concerning the size of the fetal bladder rather than the embryologic origin; it may be a single or multiple malformation; the possible association with the bowel and/or encephalic malformations will decide the outcome and prognosis in fetal megacystis.
\end{abstract}

Keywords: immunohistochemistry; urinary tract malformations; megacystis; enteric nervous system; outcome and prognosis

\section{Introduction}

Congenital anomalies of the kidney and urinary tract (CAKUT) are the most common congenital malformations, with a frequency of 3-6 per 1000 live births. They include: isolated kidney malformations (agenesis, hypo-dysplasia, multicystic renal disease, ureteropelvic junction obstruction) and urinary tract malformations (megaureters, megacystis, posterior urethral valve (PUV), urethral atresia/obstruction, urogenital sinus and cloacal malformations, obstructive ureterocele). CAKUT have also been reported in Prune-Belly syndrome (PBS) and associated genetic syndromes, mainly 13, 18 and 21 trisomy [1].

The fetal bladder may be viewed and evaluated by ultrasound from the 12th gestational week, as a pelvic, oval, anechogen structure less than $6 \mathrm{~mm}$ of sagittal diameter.

An increased sagittal diameter of the fetal bladder has been considered as megacystis, regardless of etiologic factors and macroscopic features. Therefore, prenatal megacystis may be considered mainly an ultrasound diagnosis [1,2]. 
We aimed to evaluate the structural and histological differences between two megacystis diagnosed by routine prenatal ultrasound screening in the Obstetrical and Gynecological Clinic, University of Catania, Italy, and examined in the Pathologic Anatomy Section of G.F. Ingrassia Department, University of Catania.

\section{Materials and Methods}

Two fetuses with urinary tract malformations were examined in the Pathologic Anatomy Section of the G.F. Ingrassia Department, University of Catania, Italy. The study was conducted in accordance with with the Declaration of Helsinki, and the protocol was approved by the Ethics Committee of Catania 1 (48102 of 7 November, according to national legislation about osservational studies, 20 March 2008, AIFA).

Case 1: 19th week termination of pregnancy (TOP); male, 330 gr; total length $24 \mathrm{~cm}$; crown-rump (CR) length $15.3 \mathrm{~cm}$; medial length of foot $3 \mathrm{~cm}$; cranial circumference $15.5 \mathrm{~cm}$; thoracic circumference $13 \mathrm{~cm}$; abdominal circumference $11 \mathrm{~cm}$. Ruby red skin; perforated orifices. Umbilical cord stump $10 \times 0.7 \mathrm{~cm}$; edema of penis. The anatomic relationship of thoracic organs was normal: heart in situs solitus with atrium-ventricular and ventricular-vascular concordance and lung development coherent with gestational age. Examination of the abdominal cavity showed: megacystis (25 mm sagittal diameter) with thickened wall $(6 \mathrm{~mm})$, megaureter with stenosis of ureteral orifices in the bladder, and bilateral hydronephrotic kidneys (both $18 \mathrm{~mm}$ maximum diameter).

Ecographic diagnosis: urinary obstruction syndrome due to posterior urethral valve (PUV). The US (ultrasonographic) examination showed a megacystis with a maximum diameter of $44 \mathrm{~mm}$ and mild hydroureteronephrosis with bright hyperechogenic kidneys. The bladder had the so-called "key hole sign", suggesting a possible PUV. Fetal biometry and amniotic fluid were appropriate for gestational age (GA). No other major abnormalities were detected (Figure 1).
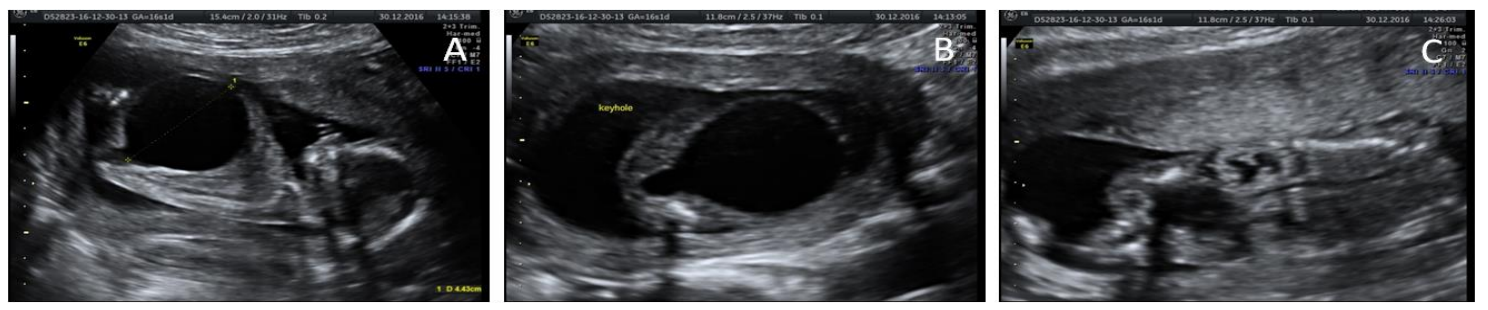

Figure 1. Case 1, US (ultrasonographic). (A) Sagittal view of the entire fetus with megacystis. (B) Coronal view of the bladder with the keyhole sign. (C) Coronal view of the hyperechogenic kidneys. Courtesy of Dr. G. Giunta.

Case 2: 15th week TOP; XXY karyotype; $40 \mathrm{gr}$; total length $11 \mathrm{~cm}$; crown-rump (CR) length $8.5 \mathrm{~cm}$; medial length of foot $1.5 \mathrm{~cm}$; cranial circumference $7 \mathrm{~cm}$; thoracic circumference $6 \mathrm{~cm}$; abdominal circumference $7 \mathrm{~cm}$. Ruby red skin; imperforated orifices. Turricephal and anencephal skull, prognathism, prominent ocular bulbs and low implant of the ears. The abdominal wall was flaccid due to the incomplete development of the diaphragm and ribs. The abdominal organs were herniated in the thoracic cavity and in the neck across the skin. Megacystis (65 mm sagittal diameter) with a thinned wall ( $2 \mathrm{~mm}$ ) occupied the abdominal and thoracic cavities; both kidneys and surrenal glands were underdeveloped (both with a maximum diameter of $10 \mathrm{~mm}$ ).

Ecographic diagnosis: Prune-Belly syndrome, PBS. The ultrasonographic (US) examination was very difficult due to maternal BMI > 35 and the anhydramnios-absence of amniotic fluid. The fetus showed a megacystis with a maximum diameter of $38 \mathrm{~mm}$. The whole fetal body had a very uncommon posture and it was impossible to assess other fetal structures because of the anhydramnios (Figure 2). 


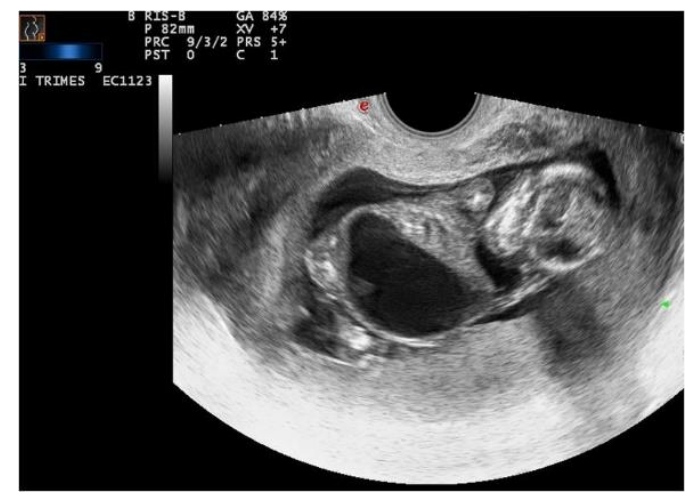

Figure 2. Case 2, US. Megacystis and anhydramnios. Courtesy of Dr. G. Giunta.

The organs of both fetuses were carefully dissected, separated from each other, fixed in $10 \%$ buffered formalin and processed until paraffin embedding. $5 \mu \mathrm{m}$ sections were stained with hematoxylin-eosin (EE). Additional immunohistochemical stainings with smooth muscular actin (SMA, Clone 1A4, Dako, Glostrup, Denmark, Dil: 1:100), S100 protein (polyclonal, Dako, Glostrup, Denmark, Dil. 1:100) and WT1c (clone WT 6F-H2, Dako, Glostrup, Denmark, prediluted) were performed on bladder and bowel slides. Bladder and bowel samples from a fetus of XXI gestational age were considered as a normal control.

\section{Results}

\subsection{Normal Control}

Bladder: SMA staining marked longitudinal and transversal muscular layers intersected by embryonal mesenchyme; nervous plexuses were evaluated with S100 protein, showing their arrangement among the muscular layers; WT1c was poorly marked in ganglion cells, neural cells and their cytoplasmic extensions (Figure 3).
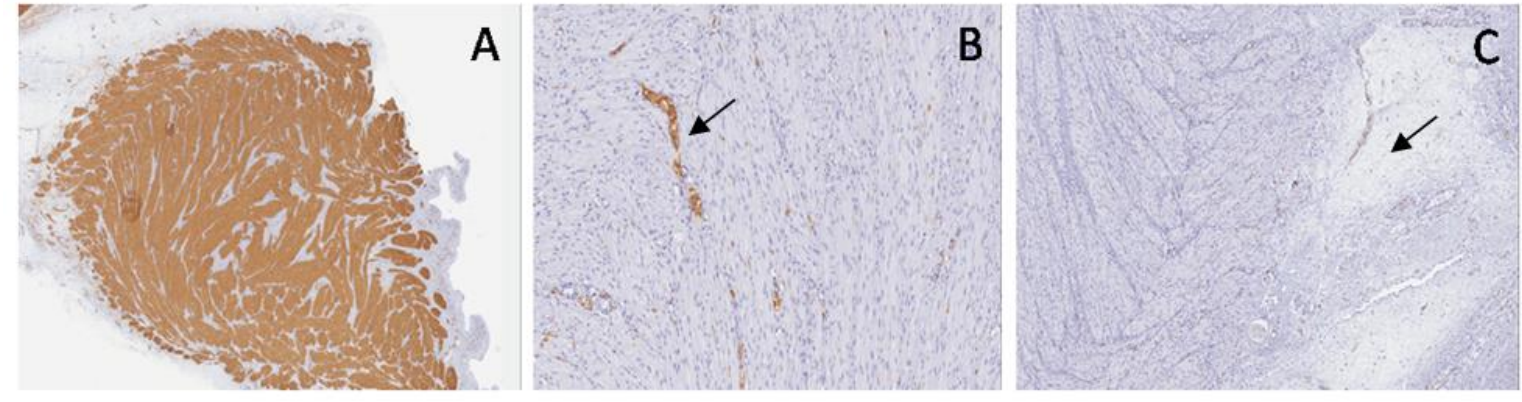

Figure 3. Normal bladder. Immunohistochemical staining shows smooth muscular actin (SMA) (A) in longitudinal and transversal muscular layers, S100 (B) in nervous plexuses (arrow) among the muscular layers and WT1c in ganglion cells (arrow) and neural cells (C).

Large and small bowel: SMA stained longitudinal and transverse muscular layers and interposed embryonal mesenchyme; S100 protein marked submucosal and myenteric plexuses with neural and ganglion cells; WT1c was shown in ganglion cells and their cytoplasmic extensions (Figure 4). 

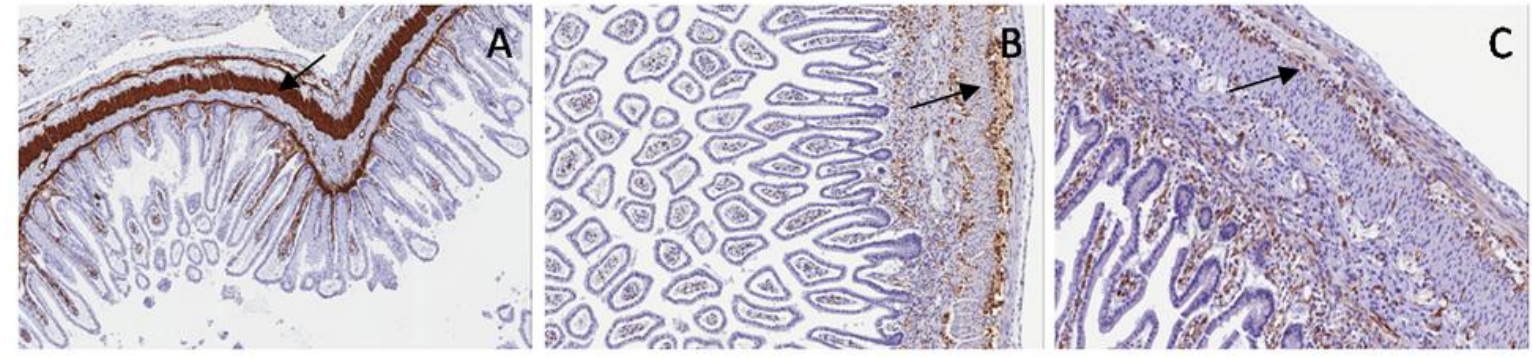

Figure 4. Normal bowel. Immunohistochemical staining shows: SMA (A) in longitudinal and transverse muscular layers (arrow), S100 (B) in submucosal and myenteric plexuses (arrow) and WT1c (C) in ganglion cells (arrow).

\subsection{Case 1 PUV}

Bladder: SMA highlighted muscular fiber disarray (shredded-carrots-like) with interposed embryonal mesenchyme; S100 staining showed hyperplastic neural plexus with irregular arrangement among muscular fibers; WT1c was poorly expressed in ganglion cells and in their cytoplasmic extensions (Figure 5).
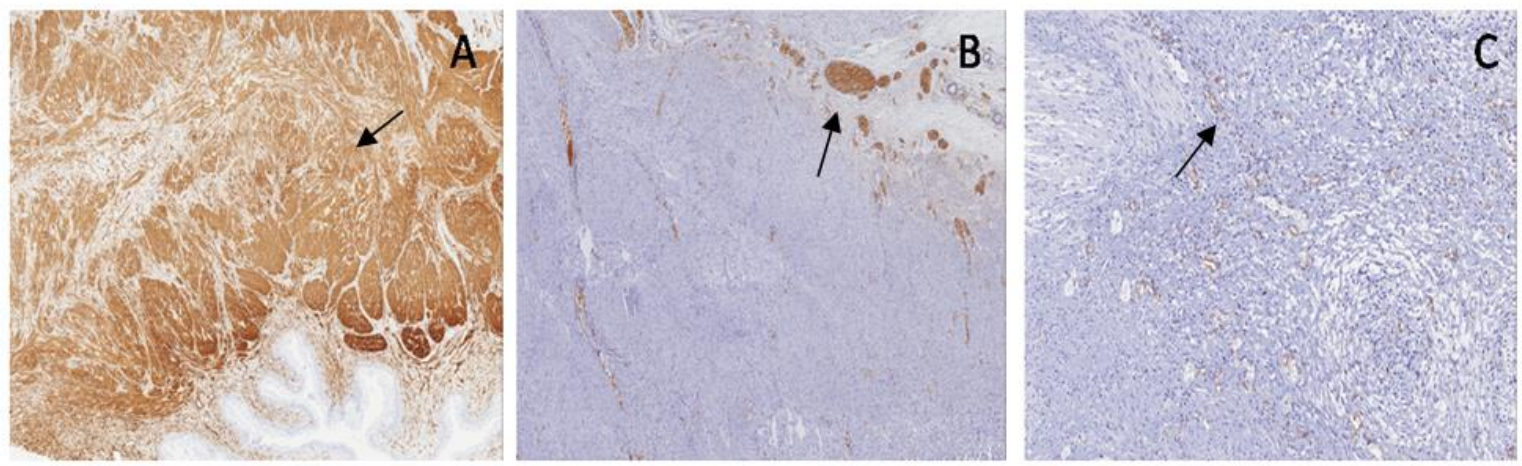

Figure 5. Case 1, posterior urethral valve (PUV). Immunohistochemical staining in bladder shows: SMA (A) in disarrayed muscular fibers (arrow), S100 (B) in hyperplastic neural plexus (arrow) and WT1c (C) poorly expressed in ganglion cells (arrow).

Large and small bowel: as in the normal control, SMA highlighted two muscular layers and interposed embryonal mesenchyme, S100 marked submucosal and myenteric plexuses with neural and ganglion cells, and WT1c was shown in ganglion cells and in their cytoplasmic extensions (Figure 6).

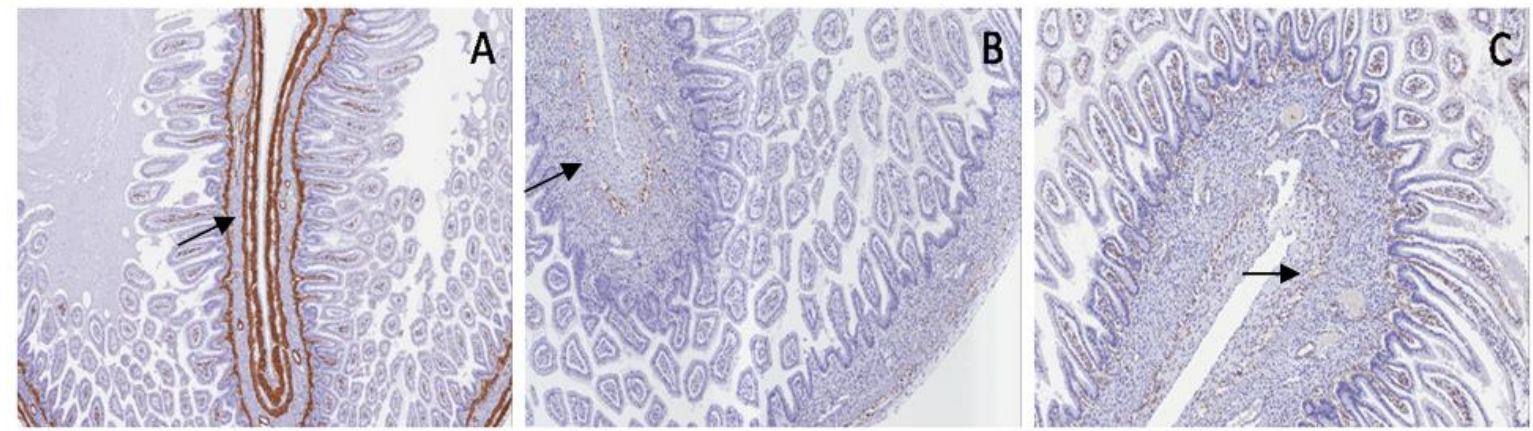

Figure 6. Case 1, PUV. Immunohistochemical staining in bowel: SMA (A) in the muscular layers (arrow), S100 (B) in submucosal and myenteric plexuses (arrow) and WT1c in ganglion cells (C) (arrow). 


\subsection{Case 2 (PBS)}

Bladder: SMA highlighted a thin, longitudinal, muscular layer with disarray of muscular fibers (shredded-carrots-like) and poor interposed embryonal mesenchyme; S100 was shown only in peripheral nervous fibers and WT1c was negative (Figure 7).
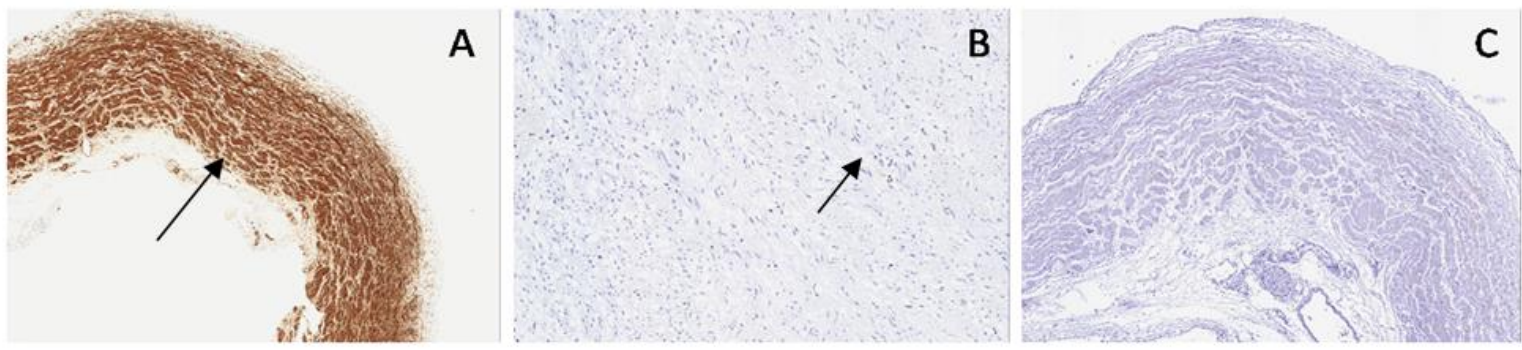

Figure 7. Case 2, Prune-Belly syndrome (PBS). Immunohistochemical staining in bladder: SMA (A) in a thin, longitudinal, muscular layer (arrow), S100 (B) only in peripheral nervous fibers (arrow), WT1c (C) negative.

Large and small bowel: SMA highlighted two longitudinal side-by-side muscular layers, without interposed embryonal mesenchyme; S100 protein and WT1c were not expressed (Figure 8).
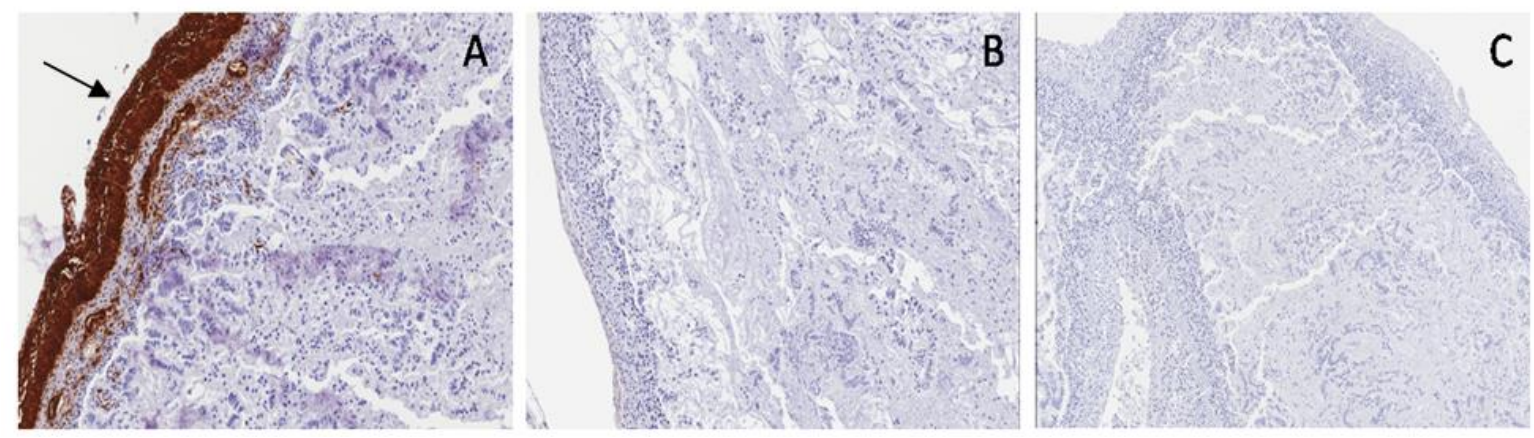

Figure 8. Case 2, PBS. Immunohistochemical staining in bowel: SMA (A) in two longitudinal side-by-side muscular layers (arrow), S100 (B) and WT1c (C) were not expressed.

\section{Discussion}

The kidney and urinary tract develops from two different embryonal sheets: kidneys, ureters and bladder trigone from the mesodermal sheet, and bladder and urethra from the endodermal sheet. The bladder develops from the fourth to seventh week of gestational age from the urogenital sinus. At the ninth week, after the involution of cloaca, the urogenital sinus opens into the amniotic cavity. CAKUT represent up to $20 \%-30 \%$ of all major congenital pre and postnatal defects [3], including both sporadic and familial cases and associated anomalies, such as syndromic malformations.

Among all CAKUT, our paper focuses on mainly bladder and urethral malformations. Isolated bladder anomalies, such as bladder agenesis, complete/incomplete duplication or bladder extrophy, are rare. Instead, morphologic bladder anomalies, known as megacystis, are more frequent and related to urethral or neuromuscular anomalies.

Fetal bladder is defined as megacystis if its longitudinal diameter is $>10 \%$ of crown-rump length in different gestational ages. In the first trimester, the longitudinal diameter may be more than $6 \mathrm{~mm}$.

Prenatal detection of a larger bladder could suggest an outlet obstruction, mainly due to urethral obstruction, but also other congenital complex malformations of the kidney and urinary tract. Moreover, in the case of megacystis, microcolon syndrome as well as neuro-muscular malformations should be considered. 
PUV has been considered the most common cause of urethral obstruction in newborn males, while obstruction of the anterior urethral valve is less common and its complications are less severe than PUV.

Three types of PUV have been described: type 1, the most common (95\%), with two mucosal folds from the bottom of veromontanum to the membranous urethra; type 2, with mucosal folds extending along posterolateral urethral wall from the ureteric orifice to the veromontanum; type 3 , with a circular diaphragm with a central opening in the membranous urethra. In any type of urethral obstruction, the high intravesical pressure leads to defects in the muscular differentiation, with fibrotic tissue interposed among muscular fibers and a thickened bladder wall [1,2].

PBS occurs mainly in baby or infant males $(97 \%)$ and is characterized by atrophy of the anterior abdominal wall due to muscular absence, anomalies in the urinary tract, such as megaureters and bilateral hydronephrosis, and testicular agenesis or cryptorchidism. More often, the bladder shows thickened walls with dilated ureteral orifices in the vesical trigone and vesicoureteral reflux. However, bladder histology is variable, showing both increased and decreased muscular fibers, with or without interposed connective tissue. In a previous study, Volmar et al. [4] described PBS with increased muscular thickness of the bladder in a case of intravesical obstruction, while a more recent work [5] reported PBS with decreased muscular thickness of the bladder in a case of urethral obstruction. In the latter case, the higher intravesical pressure, together with atrophy of the abdominal wall, could cause decreased muscular thickness, increased fibroblastic activity and overproduction of collagen type I, inhibiting muscular contractility and electrical impulse diffusion through the muscular layers.

Megacystis/megaureter syndrome is characterized by megacystis with thinned walls, vesicoureteral reflux, bilateral hydroureters and hydronephrosis and, often, dysplastic kidneys. It is frequently associated with microcolon and functional obstruction of the gastrointestinal and urinary tracts [6].

However, gestational age and vesical longitudinal diameter may be considered the main prognostic factors: it has been reported [2] that mild megacystis $(8-12 \mathrm{~mm})$ in early pregnancy (10-14 weeks) could spontaneously resolve, while severe megacystis $(>17 \mathrm{~mm})$ had a poor prognosis at any gestational age. Moreover, the ultrasound "keyhole sign", which is more frequently but not exclusively linked to PUV, has been considered the only important discriminant criteria. Some surgical therapies have been suggested to treat fetal megacystis, including amnioinfusion, vescicoamniotic shunting and vescicocentesis, but their outcome has been considered uncertain because fetal megacystis is often associated with other adverse prognostic factors.

Our two cases showed different gestational age (19 and 15 weeks), severe megacystis ( $25 \mathrm{~mm}$ and $85 \mathrm{~mm}$ ) and, above all, different development of the muscular layers (thickened and thinned muscular layers).

Due to the heterogeneity in the definition of megacystis, in bladder development evaluation, and in future outcomes, we proposed to evaluate the histological differences and potential relationship with enteric nervous system development.

The immunohistochemical expression of S100 protein, SMA and WT1c was assessed and their expression in the bladder and in the small and large bowel from normal fetus and fetuses with megacystis were compared.

Comparing normal bladder with the bladder in PUV, SMA staining highlighted a disarray of muscular fibers and increased embryonal mesenchyme, due to the higher intravesical pressure, while in PBS, it showed thinned muscular layers with only longitudinal fibers and poor interposed embryonal mesenchyme (Figure 9).

To our knowledge, for the first time, the distribution of neural components among muscular fibers using S100 protein and WT1c has been assessed in the bladder [7].

In the normal bladder, S100 protein showed small ganglionic plexuses running parallel to the muscular fibers. In PUV, S100 staining showed small and giant hyperplastic ganglionic structures running parallel to the muscular fibers, while in PBS, S100 was negative (Figure 10). 

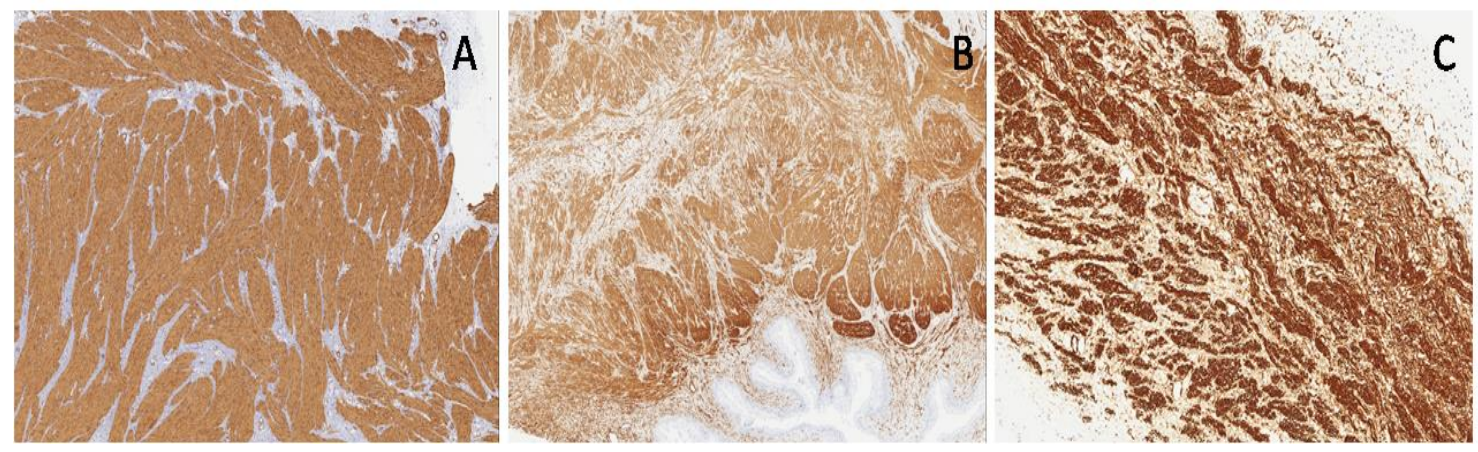

Figure 9. Comparative SMA expression in normal bladder (A), PUV (B) and PBS (C).
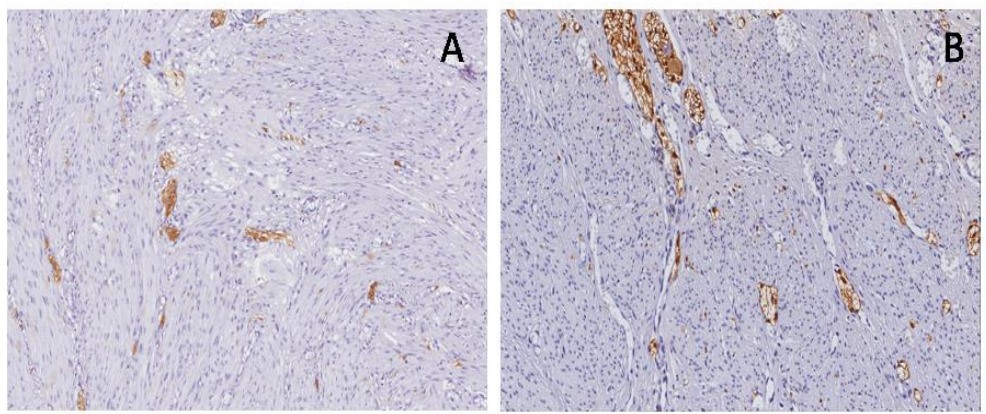

Figure 10. Comparative S100 expression in normal bladder (A), PUV (B) and PBS (C).

As concerns WT1c expression, normal bladder and PUV showed poor positivity in ganglion cells, in neural cells and in their cytoplasmic extensions, while WT1c was negative in PBS (Figure 11).
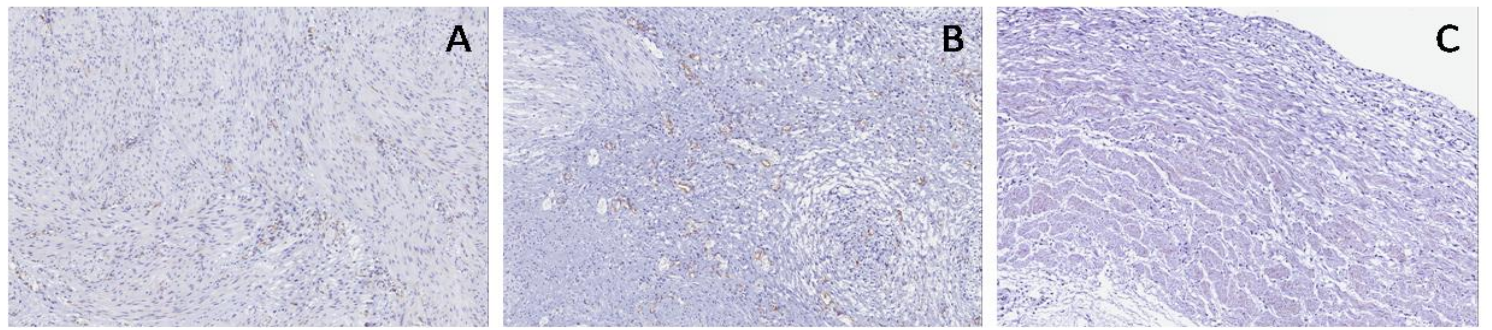

Figure 11. Comparative WT1c expression in normal bladder (A), PUV (B) and PBS (C).

Considering the relationship between the enteric nervous system and urinary tract development [6], SMA, S100 and WT1c expressions were also assessed in the enteric wall of the fetuses in order to to prove or deny their relationship and, above all, the pre or postnatal outcome as well as life expectancy.

In the normal fetus and PUV, SMA showed a double muscular layer and muscularis mucosae with interposed embryonal mesenchyme; in PBS, a single thickened muscular layer and disrupted muscularis mucosae are shown (Figure 12).

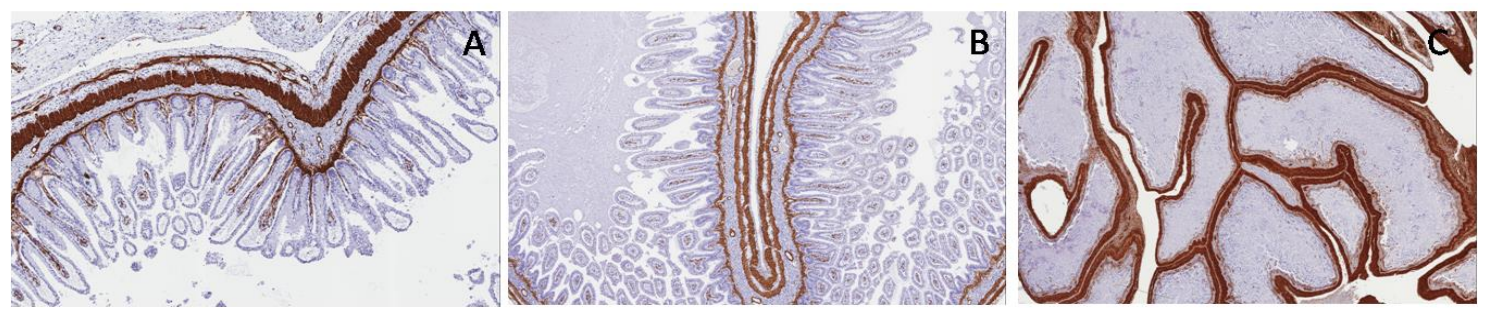

Figure 12. Comparative SMA expression in normal bowel (A), PUV (B) and PBS (C). 
S100 and WT1c expression are shown in myenteric and submucosal plexuses in the normal bowel and in PUV; both antibodies were not expressed in PBS, proving the same neuromuscular defect in the bladder and bowel (Figures 13 and 14).
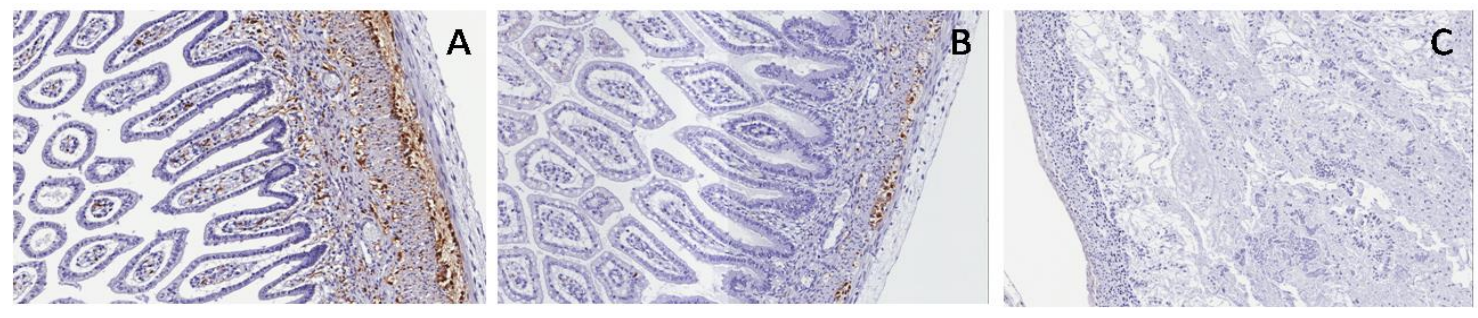

Figure 13. Comparative S100 expression in normal bowel (A), PUV (B) and PBS (C).
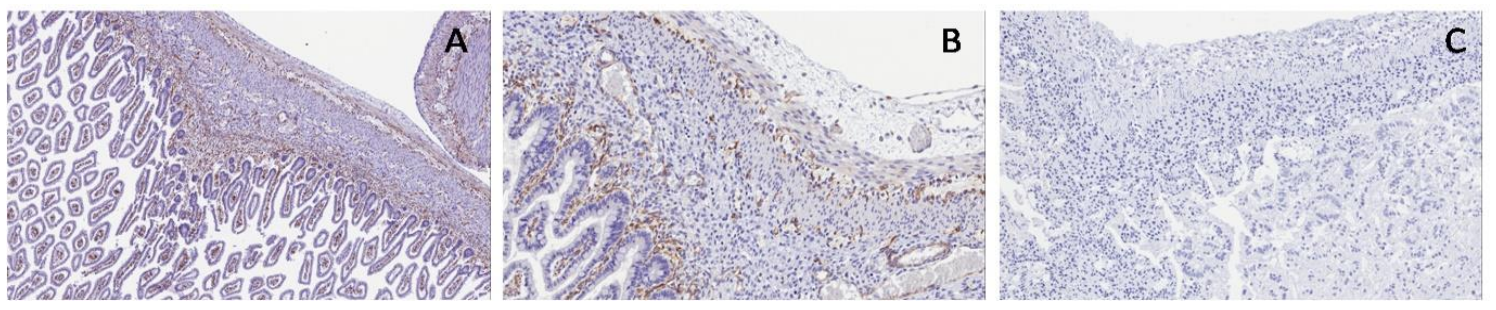

Figure 14. Comparative WT1c expression in normal bowel (A), PUV (B) and PBS (C).

Recent literature reports interesting data about the relationship between the intrinsic enteric nervous system (ENS) and the central nervous system, communicating through the gut-brain axis. Both systems develop from the neural crest progenitor and are regulated by the interactions among enteric neurons, glia and enteric-endocrine cells [8]. We hypothesized the same embryologic nature in enteric and vesical neural plexuses. Therefore, S100 and WT1c expression were evaluated in bladder and bowel muscular layers. Both markers were not expressed in the bladder and bowel of PBS associated with anencephaly, confirming a close relationship between encephalic and peripheral neural development.

In conclusion, megacystis could be considered only a macroscopic definition, concerning the size of the fetal bladder rather than the embryologic origin [9]. A larger bladder may be a single malformation or multiple malformations associated with the bowel and/or encephalic malformations, which decide the outcome and prognosis in fetal megacystis.

Author Contributions: Conceptualization, L.P., L.S. and G.G.; Data curation, R.C., A.C., L.S.; Methodology, L.P., L.S., G.G.; Resources, A.C. and R.C.; Writing—original draft, L.P.; Writing-review \& editing, L.S. and R.C.

Funding: This research received no external funding

Acknowledgments: We wish to thank the Scientific Bureau of the University of Catania for language support.

Conflicts of Interest: The authors declare no conflict of interest.

\section{References}

1. Khong, Y.T.; Malcomson Roger, D.G. The urinary system. In Keeling's Fetal and Neonatal Pathology, 5th ed.; Springer Verlag: Berlin, Germany, 2015; pp. 643-651.

2. Taghavi, K.; Sharpe, C.; Stringer, M.D. Fetal megacystis: A systematic review. J. Pediatr. Urol. 2017, 13, 7-15. [CrossRef] [PubMed]

3. Rosenblum, S.; Pal, A.; Reidy, K. Renal development in the fetus and premature infant. Semin. Fetal Neonatal Med. 2017, 22, 58-66. [CrossRef] [PubMed] 
4. Vollmar, K.E.; Fritsch, M.K.; Perlman, E.J.; Hutchins, G.M. Patterns of congenital lower urinary tract obstructive uropathy: Relation to abnormal prostate and bladder development and the prune belly syndrome. Pediatr. Dev. Pathol. 2001, 4, 467-472. [CrossRef] [PubMed]

5. Julio Junior Helce, R.; Costa Suelen, F.; Costa Waldemar, S.; Barcellos Sampaio, F.J.; Favorito Luciano, A. Structural study of the bladder in fetuses with prune belly syndrome. Neurourol. Urodyn. 2017, 37, 148-152. [CrossRef]

6. Hofmann, A.D.; Duess, J.W.; Puri, P. Congenital anomalies of the kidney and urinary tract (CAKUT) associated with Hirschsprung's disease: A systematic review. Pediatr. Surg. Int. 2014, 30, 757-761. [CrossRef]

7. Parenti, R.; Salvatorelli, L.; Musumeci, G.; Parenti, C.; Giorlandino, A.; Motta, F.; Magro, G. Wilms'tumor 1 (WT1) protein expression in human developing tissues. Acta Histochem. 2015, 117, 386-396. [CrossRef]

8. Kulkarni, S.; Ganz, J.; Bayrer, L.; Bogunovic, M.; Rao, M. Advances in enteric neurobiology: The "brain" in the gut in health and disease. J. Neurosci. 2018, 38, 9346-9354. [CrossRef] [PubMed]

9. Fontanella, F.; Maggio, L.; Verheij, J.B.G.M.; Duin, L.K.; Adama Van Scheltema, P.N.; Cohen-Overbeek, T.E.; Pajkrt, E.; Bekker, M.; Willekes, C.; Bax, C.J.; et al. Fetal megacystis: A lot more than LUTO. Ultrasound Obstet. Gynecol. 2019, 53, 779-787. [CrossRef] [PubMed]

(C) 2019 by the authors. Licensee MDPI, Basel, Switzerland. This article is an open access article distributed under the terms and conditions of the Creative Commons Attribution (CC BY) license (http://creativecommons.org/licenses/by/4.0/). 